\title{
Search for New Resonances
}

\author{
Aytul Adiguzel, on behalf of the ATLAS and CMS collaborations
}

Istanbul University, Istanbul, Turkey

\begin{abstract}
Many theories beyond the Standard Model (BSM) predict new phenomena at the highest energies accessible by the LHC. Several searches for new resonances have been performed by the ATLAS and CMS experiments. This paper presents results using $13 \mathrm{TeV} p p$ data collected during Run 2 by the ATLAS and CMS experiments.

Keywords: LHC, ATLAS, CMS, Exotic, Resonances

DOI: 10.31526/ACP.NDM-2020.13

\section{INTRODUCTION}

New particles appear in a variety of SM extensions such as supersymmetry, grand unification models and extra dimensions. Looking for these particles is a good way for testing the models. It is also important to perform model-independent searches, as we never know what new physics might be out there. Resonance searches are the classic collider methodology in searches for new particles and their excitations. The majority of exotic searches follow this methodology. There has been a great variety of recent searches for new resonances from the ATLAS [1] and CMS [2] Collaborations at the LHC [3]. Some searches are summarized in this paper. They are grouped according to the final states considered, either hadronic or leptonic. Results are obtained using $\sim 36, \sim 80$ or $\sim 140 \mathrm{fb}^{-1}$ of $13 \mathrm{TeV} p p$ collision Run 2 data.
\end{abstract}

\section{HADRONIC FINAL STATES}

\section{Search for high mass dijet resonances with a new background prediction method:}

A search for narrow and broad resonances decaying to a pair of jets has been performed by the CMS Collaboration [4]. The search uses $p p$ data corresponding to an integrated luminosity of $137 \mathrm{fb}^{-1}$. Spatially close jets are combined into 'wide jets' and used to determine the dijet mass, as introduced for CMS dijet searches in Ref [5]. There are two methods used for background estimation. The ratio method is a new data-driven method which predicts the background from a control region (CR) where the pseudorapidity separation of the two jets, $\left|\Delta \eta_{j j}\right|$, is large. This is used for $m_{j i}>2.4 \mathrm{TeV}$. The fit method is used in the signal region, defined by requiring $\left|\Delta \eta_{j j}\right|<1.1$. It is used for $m_{j j}<1.5 \mathrm{TeV}$. Figure 1 (a) shows the dijet mass spectrum. It also shows the background prediction from the fit and ratio methods. The different limits for $q q, q g$ and $g g$ resonances, which originate from differences in their line shapes are shown in Figure 1(b). The limits on dark matter mediators are calculated as a function of the different variables such as resonance mass and width, and on the associated coupling strength as a function of the mediator mass. A dark matter candidate of mass $1.8 \mathrm{TeV}$ and width $1 \%$ of its mass or higher is excluded at $95 \%$ confidence level (CL). A similar search is performed by the ATLAS Collaboration [6]. Excited quarks $q^{*}$ with masses below $6.7 \mathrm{TeV}$ and $Z^{\prime}$ masses below $2.7 \mathrm{TeV}$ (for the SSM $Z^{\prime}$ model ) are excluded at $95 \%$ CL using $139 \mathrm{fb}^{-1}$.

\section{Search for dijet resonances in events with an isolated lepton:}

This search focuses on a generic search for BSM resonances in the dijet invariant mass distribution constructed from events with at least one isolated lepton ( $\mu$ or $e$ ) using $79.8 \mathrm{fb}^{-1}$ of data collected by the ATLAS detector [7]. Single lepton triggers (either singlemuon or single-electron trigger) have been used to record candidate events. The anti- $k_{\mathrm{T}}$ algorithm with a distance parameter of $R=0.4$ is used to form jets. The background is modelled with a five parameter function by using a sliding window fit procedure. An example of exclusion limits for the signal models $\rho_{\mathrm{T}} \rightarrow W \pi_{\mathrm{T}} \rightarrow l v q \bar{q}$ (where a technirho decays to a $\mathrm{W}$ boson and a technipion) and $W^{\prime} \rightarrow W Z^{\prime} \rightarrow l v q \bar{q}$ is shown in Figure 1 (c). A signal for $\rho_{\mathrm{T}} \rightarrow W \pi_{\mathrm{T}}$ is excluded below $0.5 \mathrm{TeV}$, while $W^{\prime} \rightarrow W Z^{\prime}$ is excluded below a $Z^{\prime}$ mass of $2 \mathrm{TeV}$.

\section{Search for low-mass resonances decaying into two jets and produced in association with a photon:}

This search is performed to look for resonances in events containing a dijet and a high- $p_{\mathrm{T}}$ photon in the final state using $79.8 \mathrm{fb}$ of data collected by the ATLAS detector [8]. Two variants are used: one which makes no jet flavour requirements and one which requires both jets to be tagged as $b$-jets. Candidate events are collected via either a single-photon trigger or a combined trigger requiring additional jets. A search for resonances with masses from $225 \mathrm{GeV}$ to $450 \mathrm{GeV}$ is performed using the single-photon trigger while a search for resonances with masses from $450 \mathrm{GeV}$ to $1.1 \mathrm{TeV}$ is performed using combined trigger. At least one photon candidate and two jets are required in the event. Events are separated into four categories: two categories are constructed with 


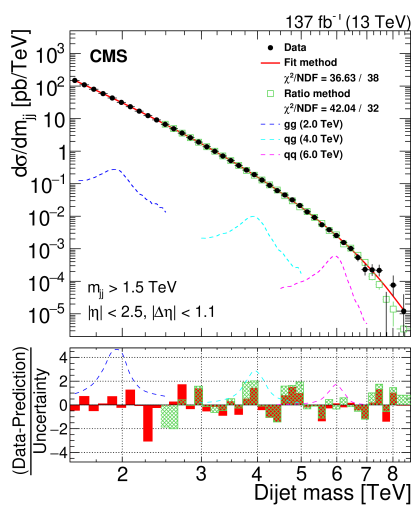

(a)

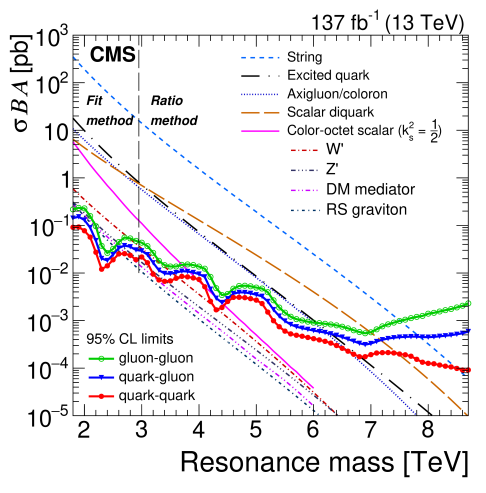

(b)

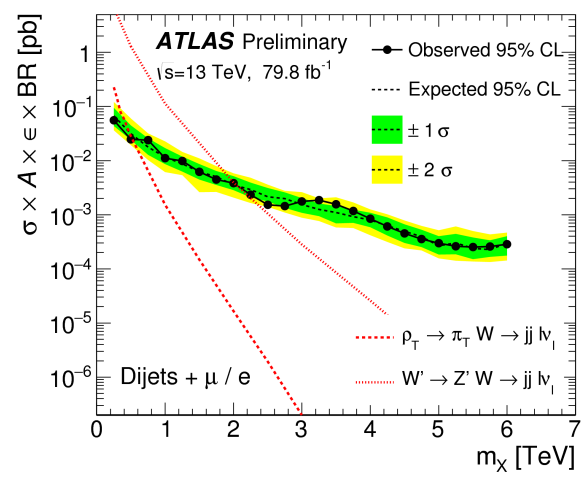

(c)

FIGURE 1: (a) Dijet mass spectrum in the signal region (points) compared to a fitted parameterization of the background (solid line) and the one obtained from the control region (green squares) (b) The observed 95\% CL upper limits on the product of the cross section, branching ratio, and acceptance for quark-quark, quark-gluon, and gluon-gluon type dijet resonances [5] (c) The 95\% CL upper limits on cross section $(\sigma)$ times acceptance $(A)$, efficiency $(\epsilon)$ and branching ratio (BR) for a Gaussian signal with a width of $15 \%$ of the mass. It also shows examples of exclusion of the $W^{\prime} \rightarrow W Z^{\prime}$ and $\rho_{\mathrm{T}} \rightarrow W \pi_{\mathrm{T}}$ models [7].

flavor-inclusive criteria, for which $b$-tagging results are ignored. The remaining two categories consist of events with $2 b$-tagging criteria. The sliding window fit procedure is applied for background estimation. Limits are set on the new-physics contributions to the $m_{j j}$ distributions, using two models: $Z^{\prime}$ axial-vector dark-matter mediators and a generic Gaussian-shaped signal contribution. Figure 2 shows the limits obtained for discrete set of points in the $g_{q^{-}} m_{Z^{\prime}}$. The results extend the current ATLAS constraints on dijet resonances to the mass range between 225 and $1100 \mathrm{GeV}$.

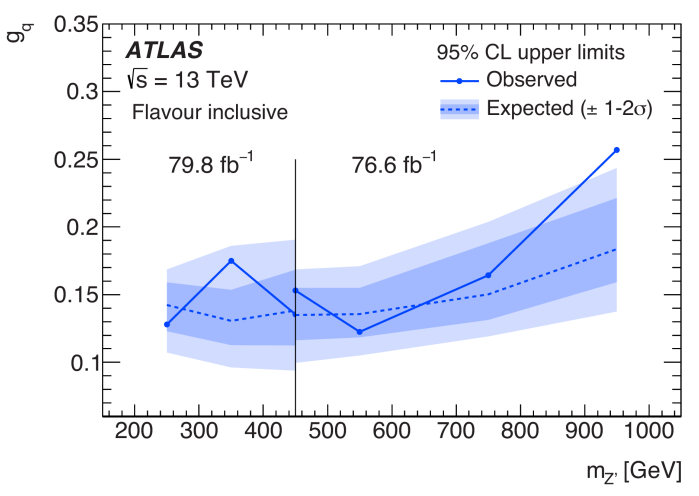

(a)

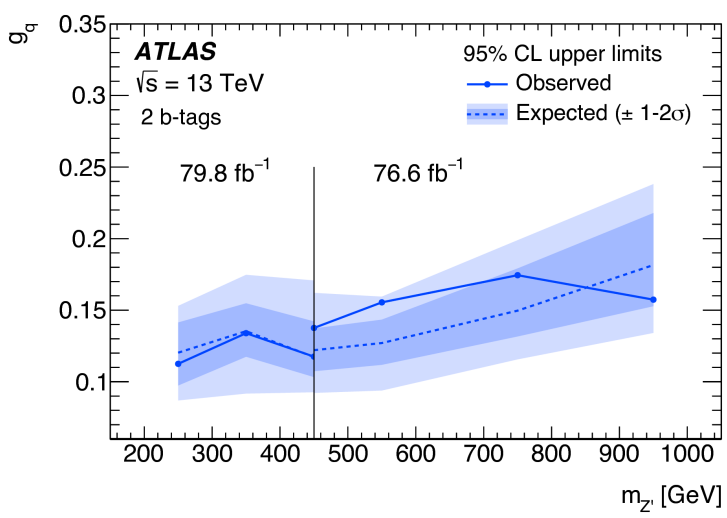

(b)

FIGURE 2: Excluded values of the coupling between $Z^{\prime}$ and quarks, at $95 \% \mathrm{CL}$, as a function of $m_{Z^{\prime}}$, from (a) the flavour-inclusive and (b) the $b$-tagged categories [8].

\section{Search for boosted resonances decaying to two b-quarks and produced in association with a jet:}

A search for new resonances decaying into a pair of bottom quarks in the merged regime is performed using $80.5 \mathrm{fb}^{-1}$ of data collected by the ATLAS detector [9]. An extra jet with high transverse momentum is required for the resonance to be merged. The mass range considered in the analysis is from $70 \mathrm{GeV}$ to $230 \mathrm{GeV}$. The reconstruction of large- $\mathrm{R}$ jets with $b$-tagging applied to the associated small-R jet is used in the analysis. The non-resonant dijet process is the dominant background contribution in the signal region (SR). A data-driven method is used by fitting the invariant mass distribution of the large-R jet $\left(m_{J}\right)$ in the SR with a parametic function for its estimate. The method is also employed by validating the procedure using the data in the $C_{\mathrm{QCD}}$. Figure 3 (a) shows the comparison to data after the marginalisation of the nuisance parameters. Limits are set on signals from dark matter mediators that decay democratically to quarks, with masses between 100 and $200 \mathrm{GeV}$ at $95 \% \mathrm{CL}$ as shown in Figure 3 (b). There is no evidence of a significant excess above the expected SM processes and limits are set on leptophobic $Z^{\prime}$ bosons with democratic axial couplings to all quark generations $\left(g_{q}\right)$ in the probed mass range. A similar search is performed by the CMS Collaboration [10]. 
The analysis has been presented using $35.9 \mathrm{fb}^{-1}$ of data. The production through gluon fusion of a scalar (pseudoscalar) decaying to $b \bar{b}$ is excluded at a resonance mass of $175 \mathrm{GeV}$, corresponding to an upper limit on $g_{q \phi}\left(g_{q z}\right)$ of 3.9 (2.5).

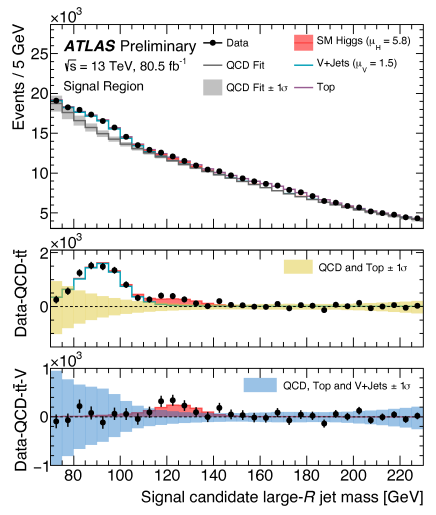

(a)

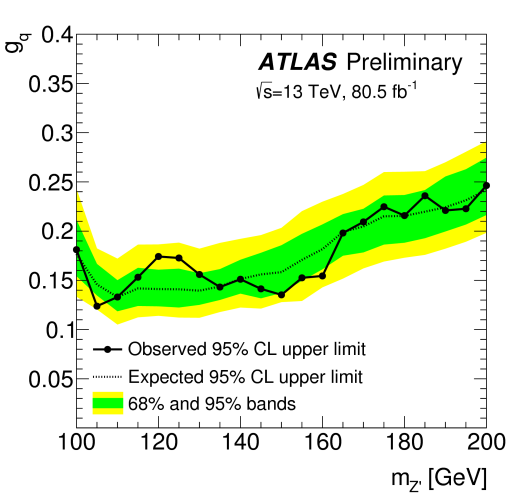

(b)

FIGURE 3: (a) Postfit plot of the SM Higgs boson, V+jets, $t \bar{t}$ and QCD fit comparison to data, (b) upper limits obtained from the invariant mass distribution on the $g_{q}$ parameter that controls the decay width of the DM mediator into SM particles [9].

\section{Search for diboson resonances in hadronic final states:}

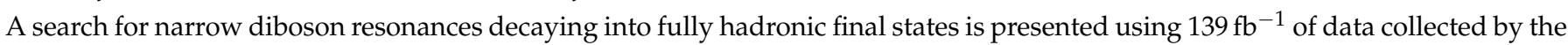
ATLAS detector [11]. There are models predicting heavy resonances decaying to SM $W / Z$ bosons. Three specific benchmark models are used: a spin-0 radion [12] decaying into WW or ZZ; a spin-1 Heavy Vector Triplet (HVT) Model [13] that provides signals such as $W^{\prime} \rightarrow W Z$ and $Z^{\prime} \rightarrow W W$; and a spin-2 graviton $G_{\mathrm{KK}} \rightarrow W W$ or $Z Z$, corresponding to Kaluza-Klein (KK) modes [14, 15] of the Randall-Sundrum (RS) graviton [16, 17, 18]. The production mechanisms of these models occur either via gluon-gluon fusion or quark-antiquark annihilation. A similar strategy as for the di-jet search is used, but boson-tagging is also applied. Boson-tagging performance is very critical to this analysis. The use of the three variables (jet mass, $D_{2}, n_{t r k}$ ) gives the largest sensitivity gain, where $D_{2}$ is defined as a ratio of two-point to three-point energy correlation functions and $n_{t r k}$ is the track multiplicity. In order to provide maximum significance for the boosted vector-boson jets relative to background jets, a three dimensional (jet mass, $D_{2}, n_{t r k}$ ) tagger is optimised. The comparison of the dijet mass distribution of the selected events in the combined $W W+Z Z$ signal regions is shown in Figure 4 (a) with the expected background distribution for the background-only fits to the data. No significant deviation from the background expectation is observed. Figure 4 (b) shows the cross-section limits extracted for the different benchmark scenarios in the $W W+Z Z$ signal region. Production of a $G_{\mathrm{KK}}$ in the bulk RS model with $k / \overline{M_{\mathrm{Pl}}}=1$ is excluded in the range $1.3-1.8 \mathrm{TeV}$ at 95\% CL. A similar analysis is also performed by the CMS Collaboration [19]. In this search, upper limits on cross sections are set between 27 and $0.2 \mathrm{fb}$ for resonance masses between 1.2 and $5.2 \mathrm{TeV}$, respectively, in a narrow-width bulk graviton model.

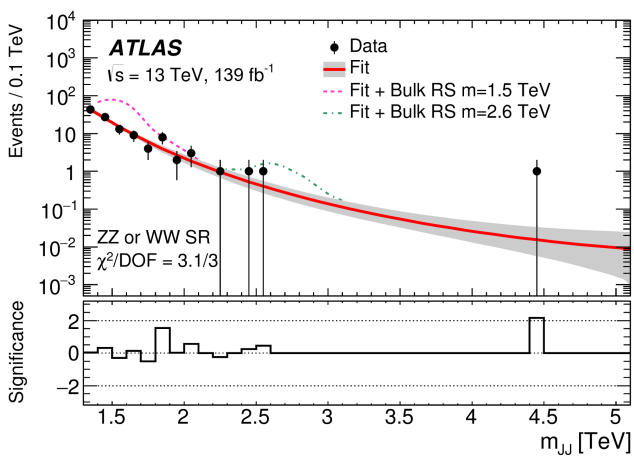

(a)

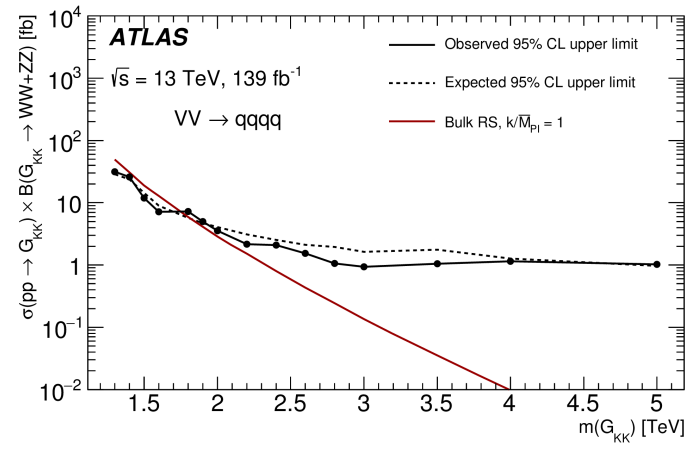

(b)

FIGURE 4: (a) Background-only fits to the dijet mass $\left(m_{j j}\right)$ distribution in the data after tagging in the combined $W W+Z Z$ signal region (b) Observed and expected limits at $95 \% \mathrm{CL}$ on the cross-section times branching ratio for $W W+Z Z$ production as a function of the Bulk RS graviton $m_{G_{K K}}[11]$. 


\section{Search for resonant $t \bar{t}$ production:}

This search presents a model-independent search for $t \bar{t}$ resonances by the CMS Collaboration [20]. This is the first search that combines all three channel topologies of the $t \bar{t}$ system by any experiment at $\sqrt{s}=13 \mathrm{TeV}$ : dilepton, single-lepton, and fully hadronic. The fully hadronic channel provides the best sensitivity along with single lepton. The decay products of the top quark become collimated because of its large Lorentz boost in the analysis and $t \bar{t}$ resonances are studied above 2 TeV. A simultaneous measurement of the backgrounds and the tag efficiency from data are performed. The data is in agreement with the background expectation for background-only hypothesis and there is no evidence for a massive $t \bar{t}$ resonance. Upper limits are set on the production cross section of heavy resonances. Limits exclude masses up to 3.8, 5.25 and $6.65 \mathrm{TeV}$ for leptophobic topcolor $Z^{\prime}$ resonances with widths of 1, 10 and 30\% respectively and up to $4.55 \mathrm{TeV}$ for Kaluza-Klein excitations of the gluon in the Randall-Sundrum model (Figure $5(\mathrm{a})$ ). So far, these are the most stringent limits on $t \bar{t}$ resonances. A similar search is also performed by the ATLAS Collaboration [21]. The $Z^{\prime}$ boson is excluded for masses up to 3.1-3.6 TeV in the topcolor-assisted-technicolor model and the Kaluza-Klein gluon is excluded for masses up to $3.4 \mathrm{TeV}$ depending on the decay widths of the particles.

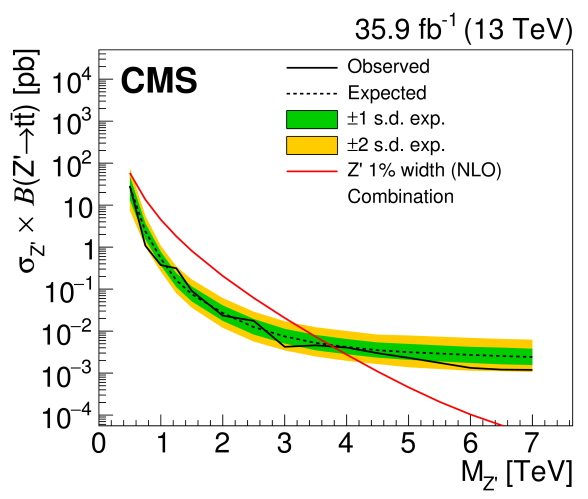

(a)

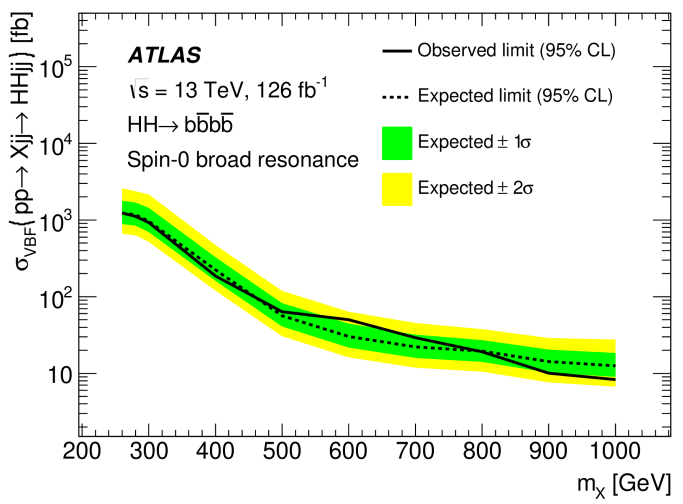

(b)

FIGURE 5: (a) Observed and expected limits at 95\% CL for Kaluza-Klein gluons [20] (b) Observed and expected 95\% CL upper limits on the production cross-section for resonant $H H$ production via VBF as a function of the mass $m_{x}$ for broad-width hypotheses [22].

\section{Search for the $H H \rightarrow b \bar{b} b \bar{b}$ process via vector boson fusion production:}

A search for Higgs boson pair production via vector boson fusion (VBF) in the $b \bar{b} b \bar{b}$ final states is performed using $126 \mathrm{fb}^{-1}$ of data collected by the ATLAS detector [22]. Events are selected using a combination of $b$-jet triggers. The background is dominated by multi-jet events, which are modelled using data-driven techniques. No significant excess is observed above the SM expectation. Limits on the production cross-section are set for a heavy resonance in the context of an extended Higgs sector. The largest deviation from the background-only hypothesis is observed at $550 \mathrm{GeV}$ with a local significance of 1.5 standard deviations. Upper limits on the production cross-section are shown in Figure 5(b) for broad-width scalar resonances at 95\% CL.

\section{Search for long-lived neutral particles decaying to displaced hadronic jets:}

A search for pair-production of long-lived neutral particles is performed using $33 \mathrm{fb}^{-1}$ of data collected by the ATLAS detector [23]. Benchmark Hidden Sector (HS) models are studied using a scalar mediator. This search focuses on the topology in which one longlived particle (LLP) decays in the inner detector (ID) and the other decays in the muon spectrometer (MS). Special techniques are employed to reconstruct the displaced tracks and vertices in the ID and MS. The background estimation is performed using data-driven methods. A scalar mediator ranges in mass from 125 to $1000 \mathrm{GeV}$, decaying into pairs of long-lived scalars ranging in mass from 8 to $400 \mathrm{GeV}$, depending on the mass of the mediator. Limits are set on the various signal mass points as shown in Figure 6(a). They are the most stringent so far on the branching ratios from the Higgs boson to several low-mass scalars, and on the cross section times branching ratio for a $200 \mathrm{GeV}$ mass $\Phi$ decaying into long-lived scalars with masses of 25 and $50 \mathrm{GeV}$.

\section{LEPTONIC FINAL STATES}

\section{Search for high-mass dilepton resonances:}

A search for heavy resonances decaying to dielectron or dimuon is performed in the mass range of $250 \mathrm{GeV}$ to $6 \mathrm{TeV}$ with the ATLAS detector [24]. The results have been updated to the full Run 2 data. At least two same-flavor leptons are required in the events. The reconstructed mass of the dilepton system $\left(m_{l l}\right)$ is required to be above $225 \mathrm{GeV}$ to avoid the $Z$ boson peak region. The analysis uses a generic signal shape to determine the significance of the observed deviations from the background estimate, and no significant deviation is seen. Upper limits are calculated on the fiducial cross-section times branching ratio to dielectrons and dimuons for generic resonances with a relative natural width in the range of zero to $10 \%$. Model dependent combined lower limits 


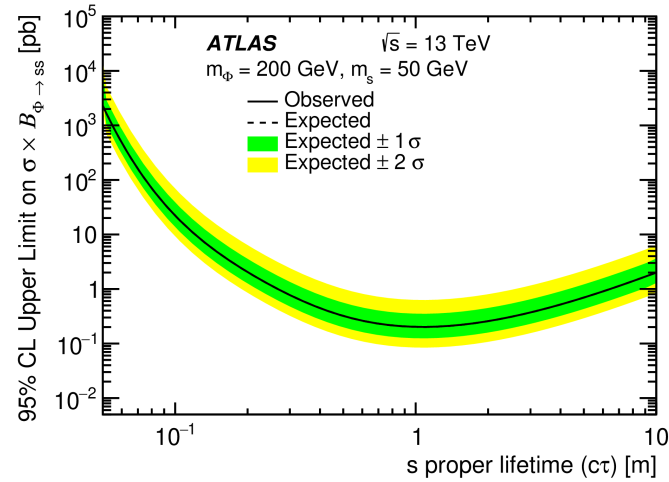

(a)

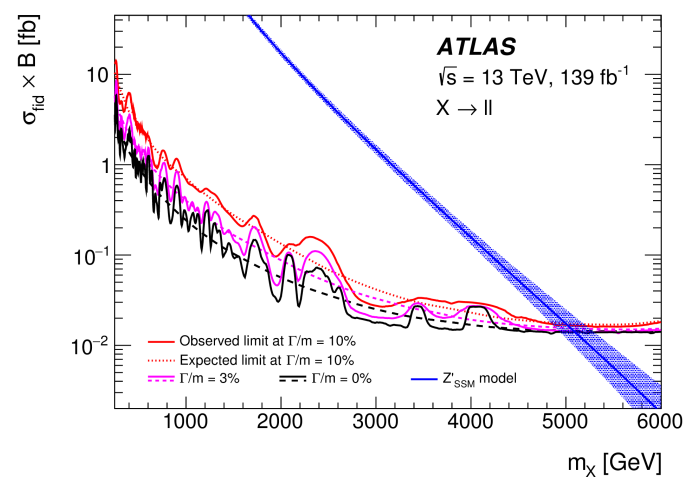

(b)

FIGURE 6: (a) 95\% CL upper limits on cross section times branching ratio for a $\Phi$ with a mass of $200 \mathrm{GeV}$ decaying to an LLP with a mass $50 \mathrm{GeV}$ [23]. (b) Upper limits at 95\% CL on the fiducial cross-section times branching ratio of dilepton resonances as a function of the pole mass for the zero, $3 \%, 10 \%$ relative width [24].

are 4.5 and $4.8 \mathrm{TeV}$ for the $E_{6}$ motivated $Z_{\psi, \chi}^{\prime}$ bosons, and $5.1 \mathrm{TeV}$ for $Z_{\mathrm{SSM}}^{\prime}$. Figure 6 (b) shows upper limits at $95 \% \mathrm{CL}$ on the fiducial cross-section times branching ratio as a function of the pole mass for the zero, $3 \%, 10 \%$ relative width, obtained using the combined dilepton channel. The theoretical cross-section for $Z_{\mathrm{SSM}}^{\prime}(\Gamma / m=3.0 \%)$ is also shown in Figure $6(\mathrm{~b})$.

\section{Search for excited leptons decaying via contact interaction to two leptons and two jets:}

This search is carried out to look for excited electrons and muons decaying via a contact interaction to final states of two leptons and two jets using $77.4 \mathrm{fb}^{-1}$ of data collected by the CMS detector [25]. This channel complements other searches for excited leptons. It has great sensitivity at large values of $M_{\ell^{*}}$ and/or $\Lambda$. Therefore it is most sensitive to heavy excited leptons. No significant excess is observed in either channel. Excited electrons (muons) up to masses of 5.6 (5.7) TeV are excluded with the usual assumption of $M_{\ell^{*}}=\Lambda$ at $95 \%$ CL (Figure 7](a)). These are the best limits so far.
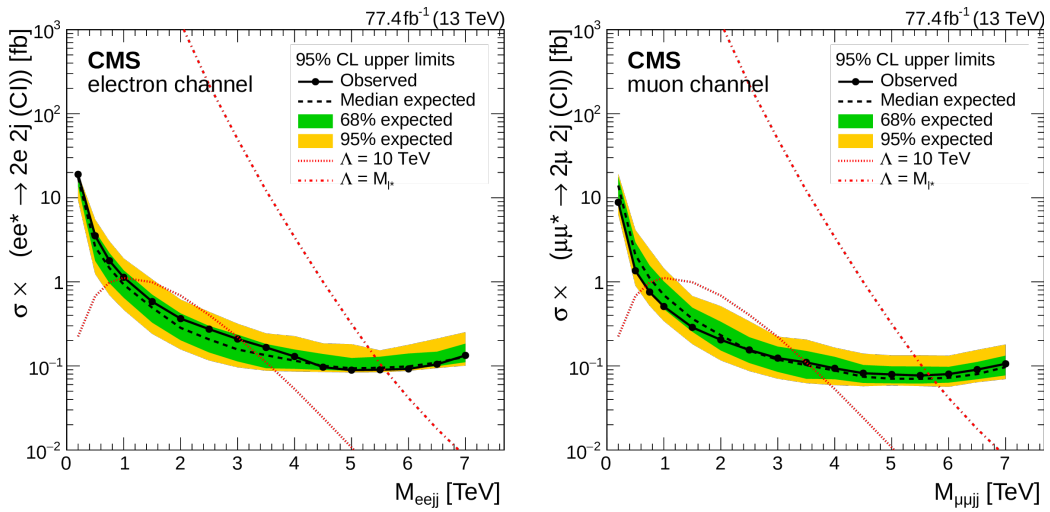

(a)

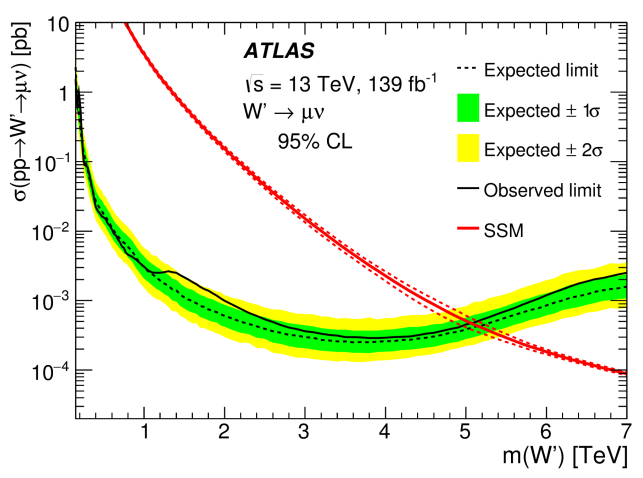

(b)

FIGURE 7: (a) Cross section limits on excited leptons using $M_{l l j j}$ distribution for the electron and muon channels. Two cases are presented: $\Lambda=M_{\ell^{*}}$ and $\Lambda=10 \mathrm{TeV}$. The cross section scales with $\Lambda$ [25]. (b) Observed and expected upper limits at the 95\% CL on $p p \rightarrow W^{\prime} \rightarrow l v$ cross section in the muon channel as a function of $W^{\prime}$ mass in the SSM [26].

\section{Search for a heavy charged boson in events with a charged lepton and missing transverse momentum:}

A heavy charged-boson resonance decaying into a charged lepton (electron or muon) and a neutrino is reported in this search using $139 \mathrm{fb}^{-1}$ of data by the ATLAS detector [26]. Events are collected using single-electron or single-muon triggers with high transverse momentum thresholds. The dominating background comes from Drell-Yan (DY) production of $W$ bosons. The transverse mass $\left(m_{\mathrm{T}}\right)$ computed from the charged lepton transverse momentum $\left(p_{\mathrm{T}}\right)$ and the missing transverse momentum $\left(E_{\mathrm{T}}^{\mathrm{miss}}\right)$ is used as the discriminating variable between signal and background. No significant excess above the SM background is observed. Upper limits on $\sigma \times B R\left(W^{\prime} \rightarrow l v\right), l=e, \mu$ are set at 95\% CL. Lower limits on the $W^{\prime}$ boson masses are $6.0 \mathrm{TeV}$ and $5.1 \mathrm{TeV}$ in the electron and muon channels, respectively, in the context of the SSM as shown in Figure 7(b). A similar search is reported by the CMS 
Collaboration using $36 \mathrm{fb}^{-1}$ [27, 28]. Values of the $W^{\prime}$ mass below $4.9 \mathrm{TeV}, 4.9 \mathrm{TeV}$ and $4.0 \mathrm{TeV}$ are excluded in the electron, muon and tau channels, respectively.

\section{Search for a heavy right-handed $W$ boson and a neutrino in events with two same-flavor leptons and two jets:}

This search presents a heavy right-handed $W$ boson $\left(W_{R}\right)$ and a heavy right-handed neutrino and a charged lepton in events with two same-flavor leptons ( $e$ or $\mu$ ) and two jets using $35.9 \mathrm{fb}^{-1}$ of data collected with the CMS detector [29]. In this search, the $W_{R}$ boson decays to a first-or-second generation charged lepton and a heavy neutrino of the same lepton flavor. Then the heavy neutrino decays to another charged lepton of the same flavor and a virtual $W_{\mathrm{R}}^{*}$. The virtual $W_{\mathrm{R}}^{*}$ decays to light quarks. For events with electrons, an unprescaled double-electron trigger is used while for the muon channel, unprescaled single-muon triggers are used. No significant excess above the Standart Model expectation is observed in the invariant mass distribution of the dilepton plus dijet system. The region in the two dimensional plane $\left(m_{W_{R}}, m_{N_{R}}\right)$ covers a large range of right-handed neutrino masses. As shown in Figure 8(a) a $W_{R}$ boson decaying into right-handed neutrino is excluded at 95\% CL up to mass of $4.4 \mathrm{TeV}$ (with a mass $\left.m_{N_{R}}=1 / 2 m_{W_{R}}\right)$. A similar search is performed by the ATLAS Collaboration using $80 \mathrm{fb}^{-1}$ of data [30]. In this search, mass values of the $W_{\mathrm{R}}$ smaller than $3.8-5 \mathrm{TeV}$ are excluded for $N_{\mathrm{R}}$ in the mass range $0.1-1.8 \mathrm{TeV}$.

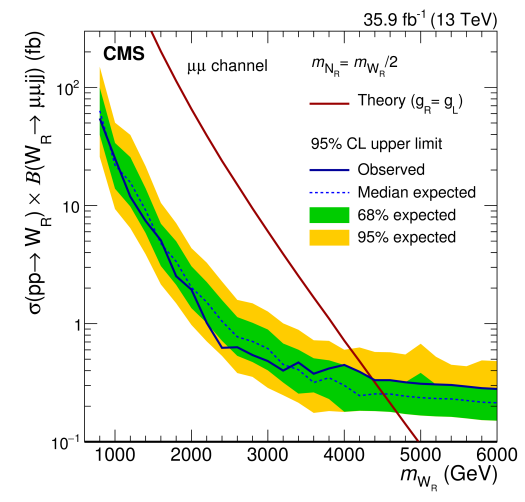

(a)

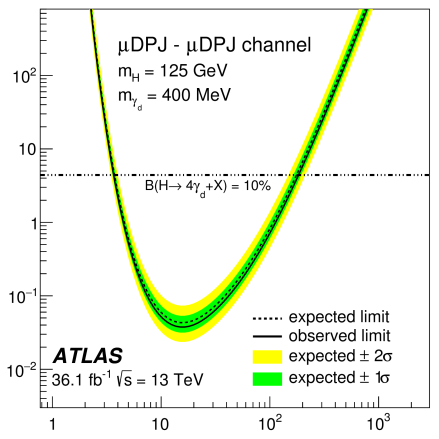

(b)

FIGURE 8: (a) Expected and observed 95\% CL upper limits on the product of the cross section and branching ratio for the muon channel [29]. (b) Upper limit at 95\% CL on the cross section times branching ratio for the processes $H \rightarrow 4 \gamma_{d}+X$ in the $(\mu \mathrm{DPJ}-$ $\mu \mathrm{DPJ}$ ) final state for $m_{H}=125 \mathrm{GeV}$ [31].

\section{Search for light long-lived neutral particles decaying into collimated leptons or light hadrons:}

Several BSM models predict the existence of dark photons. The long lived dark photons produced from the decay of a Higgs boson or heavy scalar boson and decaying into displaced collimated SM fermions is searched for using $36.1 \mathrm{fb}^{-1}$ of data collected with the ATLAS detector [31]. This analysis uses the Falkowski-Ruderman-Volansky-Zupan (FRVZ) model [32, 33] as the benchmark and a pair of dark fermions $f_{d_{2}}$ is produced via a Higgs boson $(H)$ decay. Two different cases of this model are used: the production of two or four photons. Multi-jet events are one of the main SM backgrounds in this analysis and the other sources of the backgrounds are $W+$ jets, $Z+$ jets, $t \bar{t}$, single top-quark, $W W, W Z$ and $Z Z$ events. Two dark-photon jet (DPY) classifications are considered: muonic-DPJ $(\mu \mathrm{DPJ})$ and hadronic-DPJ (hDPJ). Events are selected by the logical OR of three dedicated triggers targeting displaced objects: two muon triggers and one calorimeter trigger. Upper limits are set on the production cross section times braching ratio as a function of the proper decay length of the dark photon as shown in Figure 8 (b). A $\sigma \times B R$ above 4 pb is excluded for a Higgs boson decaying into two dark photons for dark-photon decay lengths between $1.5 \mathrm{~mm}$ and $307 \mathrm{~mm}$.

\section{Search for heavy neutral leptons produced in decays of $W$ bosons using prompt and displaced signatures:}

Heavy neutral leptons (HNLs) that are produced through mixing with muon or electron neutrinos are investigated using leptonic decays of $W$ bosons extracted from $32.9 \mathrm{fb}^{-1}$ to $36.1 \mathrm{fb}^{-1}$ of data collected with the ATLAS detector [34]. Both prompt and displaced leptonic decay signatures are reported in this search. Three leptons produced at the interaction point (IP) with a veto on sameflavor opposite-charge topologies are required to reconstruct the prompt signature. A prompt muon from the $W$ boson decay and the requirement of a dilepton vertex displaced on the transverse plane by $4-300 \mathrm{~mm}$ from the IP are required to reconstruct the displaced signature. The constraints on the HNL mixing to muon and electron neutrinos are set for HNL masses in the range $4.5-50 \mathrm{GeV}$. 


\section{SUMMARY}

ATLAS and CMS searches for BSM resonances in hadronic/leptonic final states have utilised 36-139 $\mathrm{fb}^{-1}$ of LHC Run $2 p p$ data at $\sqrt{s}=13 \mathrm{TeV}$. All the results from the experiments are accessible in Refs [35, 36]. No significant deviations from the SM background hypothesis have been observed so far. Processing of the full LHC Run $2139 \mathrm{fb}^{-1}$ data set is in progress.

\section{ACKNOWLEDGEMENTS}

A. Adiguzel acknowledges financial support from Istanbul University Scientific Research Commission (BAP) project ID 33250.

\section{References}

[1] ATLAS Collaboration, JINST 3 (2008) S08003

[2] CMS Collaboration, JINST 3 (2008) S08004

[3] L. Evans and P. Bryant (editors), JINST 3 (2008) S08001

[4] CMS Collaboration, CMS-EXO-19-012, [arXiv:hep-ex/1911.03947], https://cds.cern.ch/record/2699883

[5] CMS Collaboration, Phys. Rev. D 91, (2015) 052009. [arXiv:hep-ex/1501.04198]

[6] ATLAS Collaboration, [arXiv:hep-ex/1910.08447]

[7] ATLAS Collaboration, ATLAS-CONF-2018-015| http://cdsweb.cern.ch/record/2621126

[8] ATLAS Collaboration, Phys. Lett. B 795, (2019) 56, [arXiv:hep-ex/1901.10917]

[9] ATLAS Collaboration, ATLAS-CONF-2018-052. https://cds.cern.ch/record/2649081

[10] CMS Collaboration, Phys. Rev. D 99 (2019) 012005 |[arXiv:hep-ex/1810.11822]

[11] ATLAS Collaboration, JHEP 09, (2019) 091, [arXiv:hep-ex/1906.08589]

[12] A. Carvalho, [arXiv:hep-ph/1404.0102]

[13] D. Pappadopula, A. Thamm, R. Torre and A. Wulzer, JHEP 09, (2014) 060, [arXiv:hep-ph/1402.4431]

[14] L. Randall and R. Sundrum, Phy. Rev. Lett. 83 (1999) 3370 [arXiv:hep-ph/9905221]

[15] T. Han, J.D. Lykken and R.-J. Zhang, Phys. Rev. D 59, (1999) 105006, [arXiv:hep-ph/9811350]

[16] K. Agashe, H. Davoudiasl, G. Perez and A. Soni, Phys. Rev. D 76, (2007) 036006| [arXiv:hep-ph/0701186]

[17] O. Antipin, D. Atwood and A. Soni, Phys. Lett. B 666, (2008) 155. [arXiv:hep-ph/0711.3175]

[18] O. Antipin and A. Soni, JHEP 10, (2008) 018] [arXiv:hep-ph/0806.3427]

[19] CMS Collaboration, CMS-B2G-18-002 |[arXiv:hep-ex/1906.05977]| https ://cds . cern.ch/record/2678480

[20] CMS Collaboration, JHEP 04, (2019) 031: [arXiv:hep-ex/1810.05905]

[21] ATLAS Collaboration, Phys. Rev. D 99, (2019) 092004,[arXiv:hep-ex/1902.10077]

[22] ATLAS Collaboration, [arXiv:hep=ex/2001.05178]

[23] ATLAS Collaboration, CERN-EP-2019-240, [arXiv:hep-ex/1911.12575], http://cds .cern.ch/record/2702949

[24] ATLAS Collaboration, Phys. Lett. B 796, (2019) 68, [arXiv:hep-ex/1903.06248]

[25] CMS Collaboration, CMS-EXO-18-013, [arXiv:hep-ex/2001.04521]. https ://cds.cern.ch/record/2706282

[26] ATLAS Collaboration, Phys. Rev. D 100 (2019) 052013, [arXiv:hep-ex/1906.05609]

[27] CMS Collaboration, JHEP 06, (2018) 128, [arXiv:hep-ex/1803.11133]

[28] CMS Collaboration, Phys. Lett. B 792, (2019) 107, [arXiv:hep-ex/1807.11421]

[29] CMS Collaboration, JHEP 05, (2018) 148, [arXiv:hep-ex/1803.11116]

[30] ATLAS Collaboration, Phys. Lett. B 798, (2019) 134942][arXiv:hep-ex/1904.12679]

[31] ATLAS Collaboration, CERN-EP-2019-140, [arXiv:hep-ex/1909.01246]. http://cds .cern.ch/record/2688373

[32] A. Falkowski, J. T. Ruderman, T. Volansky and J Zupan, JHEP 05, (2010) 077. [arXiv:hep-ph/1002.2952]

[33] A. Falkowski, J. T. Ruderman, T. Volansky and J Zupan, Phy. Rev. Lett. 105, (2010) 241801.|[arXiv:hep-ph/1007.3496]

[34] ATLAS Collaboration, JHEP 10, (2019) 265, [arXiv:hep-ex/1905.09787]

[35] https://twiki.cern.ch/twiki/bin/view/AtlasPublic/ExoticsPublicResults

[36] http://cms-results.web.cern.ch/cms-results/public-results/publications/EXo/index.html 